\title{
A Novel IVPLTS Decision Method Based on Regret Theory and Cobweb Area Model
}

\author{
Peng Li $(\mathbb{D}$ and Huanhuan Peng \\ College of Economics and Management, Jiangsu University of Science and Technology, Zhenjiang, Jiangsu 212003, China \\ Correspondence should be addressed to Peng Li; jellyok@126.com
}

Received 28 May 2020; Revised 6 July 2020; Accepted 27 November 2020; Published 9 December 2020

Academic Editor: Zeshui Xu

Copyright (C) 2020 Peng Li and Huanhuan Peng. This is an open access article distributed under the Creative Commons Attribution License, which permits unrestricted use, distribution, and reproduction in any medium, provided the original work is properly cited.

\begin{abstract}
For the multiple criteria decision-making $(\mathrm{MCDM})$ problem with interval-valued probabilistic linguistic information, we propose a novel method considering the regret theory and cobweb area model. We first propose a new score function, which can be used to compare different interval-valued probabilistic linguistic term sets (IVPLTSs) and transform the IVPLTSs into crisp numbers. Some properties of the score function are verified. Then, we utilize the regret theory to obtain the perceived utilities of decision makers (DMs), which can reflect the DMs' bounded rationality. Furthermore, we use the cobweb area model to aggregate decision information. Finally, a real case of evaluating nursing homes is used to illustrate the effectiveness and features of our method.
\end{abstract}

\section{Introduction}

Multiple criteria decision-making (MCDM) widely exists in all aspects of human life. In the real decision environment, due to the complexity of decision-making problems and the limited personal knowledge of decision makers (DMs), it is difficult for DMs to express their preference information with crisp values. In 1975, Zadeh [1] first proposed the concept of fuzzy linguistic approach, advocating the use of natural linguistic instead of crisp numbers to express qualitative evaluation information in MCDM problems. Then, Rodriguez et al. [2] developed the fuzzy linguistic information and proposed the definition of hesitant fuzzy linguistic term set (HFLTS). HFLTS can express the hesitance of DMs but cannot reflect the importance of the linguistic terms. To cope with this issue, Pang et al. [3] proposed the definition of probabilistic linguistic term sets (PLTSs). Since the concept of PLTS was proposed, there had been a large amount of studies about PLTS, such as operational rules for PLTSs $[3,4]$, outranking methods [5], and preference relation of PLTSs [6].

Based on the PLTS, Bai et al. [7] further proposed the interval-valued probabilistic linguistic term set (IVPLTS) and developed some comparison rules, operation rules, and aggregation operator. Yu et al. [8] proposed a new possibility degree method for uncertain probabilistic linguistic term set (UPLTS). Zhang et al. [9] considered probability distribution and interval-valued hesitant fuzzy set and proposed the definition of probabilistic interval-valued hesitant fuzzy set (P-IVHFS). Jin et al. [10] proposed the basic operation rules and aggregation operators of uncertain probabilistic linguistic term set (UPLTS) and extended the traditional TOPSIS method to the UPLTS environment. Krishankumar et al. [11] proposed interval-valued probabilistic linguistic simple weighted geometry (IVPLSWG) to aggregate preference information of decision makers and extended the VIKOR method to the decision environment of IVPLTS.

For the aspect of behavior theory, extensive studies have been conducted. Liu and Li [12] applied prospect theory to the decision environment of probabilistic linguistic and proposed a multiobjective optimization method of MULTIMOORA based on prospect theory. Gu et al. [13] proposed a multiattribute decision-making framework based on prospect theory in probabilistic linguistic environment. Wang et al. [14] constructed a novel risk priority model for failure mode and effects analysis (FMEA). Qin [15] proposed a capital asset pricing model based on regret theory to explore the impact of regret aversion on capital pricing. Bai 
and Sarkis [16] proposed a new hybrid group decisionmaking method combining hesitant fuzzy set and regret theory for the evaluation and selection of block chain technology.

Interval-valued probabilistic linguistic term set (IVPLTS) is further improved on the basis of PLTS and can solve the problem of uncertain probability. In view of the strong applicability and advantages of IVPLTS, we extend the decision framework of IVPLTS. However, there are few studies of behavior theory in interval-valued probabilistic linguistic environment. It is necessary to consider the research of decision-makers' limited rationality. Regret theory is simpler than prospect theory [17]. Furthermore, long-term care for elders has been a serious problem in China. A rational method of evaluating nursing homes is necessary. Score function is a kind of effective tool to defuzzy probabilistic linguistic information, which can make the decision process simple. A suitable score function should be flexible and can reflect preference of DM. Therefore, in this paper, we will propose a novel decision-making method for IVPLTSs based on regret theory and apply the method to the problem of selecting nursing homes for a hospital. The main contributions of our method can be concluded as follows:

(1) We propose a new score function for IVPLTSs containing risk parameter and preference parameter of DMs. DMs can flexibly choose the two parameters according their risk attitudes and preferences and compare different IVPLTSs.

(2) We present a novel decision-making method for IVPLTSs using regret theory and cobweb area model, which can effectively reflect the bounded rationality of DMs and relieve the problem that some extremely large or small values exert too much influence on the final decision result.

The remainder of our paper is shown as follows. Section 2 reviews some basic definition and operational rules of IVPLTSs and regret theory. In Section 3, a novel score function for IVPLTSs is presented. Section 4 puts forward a novel decision method based on regret theory and cobweb area model. Section 5 applies our proposed method to a real case study and compares with the traditional TOPSIS method to illustrate the effectiveness and traits of our method. Section 6 makes a summary of our method and presents the future research scope.

\section{Preliminaries}

2.1. Interval-Valued Probabilistic Linguistic Term Set. In our real life, DMs usually use linguistic information to express their opinions rather than crisp numbers. For example, we can use "good" or "poor" to describe the quality of a car. The definition of linguistic term set (LTS) can be shown as follows.
Definition 1 (see [18]). Let $\tau$ be a positive integer, a symmetrical LTS can be defined as $S=\left\{s_{\alpha} \mid \alpha=-\tau, \ldots\right.$, $-1,0,1, \ldots, \tau\}$, where $s_{\alpha}$ is called linguistic term.

The basic operational rules for LTSs can be concluded as follows [18].

Definition 2 (see [18]). Let $s_{\alpha_{1}}$ and $s_{\alpha_{2}}$ be two linguistic terms and $\lambda$ be a positive number; then, the following rules hold:
(1) $s_{\alpha_{1}} \oplus s_{\alpha_{2}}=s_{\alpha_{1}+\alpha_{2}}$
(2) $s_{\alpha_{1}} \otimes s_{\alpha_{2}}=s_{\alpha_{1} \times \alpha_{2}}$
(3) $\lambda s_{\alpha_{1}}=s_{\lambda \alpha_{1}}$
(4) $\left(s_{\alpha_{1}}\right)^{\lambda}=s_{\alpha_{1}^{\lambda}}^{\lambda}$

Pang et al. [3] proposed the definition of probabilistic linguistic term set (PLTS) as follows.

Definition 3 (see [3]). Let $S=\left\{S_{t} \mid=-\tau, \ldots,-1,0,1, \ldots, \tau\right\}$ be a LTS, a PLTS on $S$ can be defined as $L(p)=\left\{L^{(k)}\right.$ $\left.\left(p^{(k)}\right) \mid L^{(k)} \in S, p^{(k)} \geq 0, k=1,2, \ldots, \# L(p), \sum_{k=1}^{\# L(p)} p^{(k)}\right\}$, where $L^{(k)}\left(p^{(k)}\right)$ is the linguistic term $L^{(k)}$ with respect to probability $p^{(k)}$ and $\# L(p)$ is the number of different LTSs in $L(p)$.

Bai et al. [7] extend the PLTS to interval-valued probabilistic linguistic term set (IVPLTS) as follows.

Definition 4 (see [7]). Let $S=\left\{S_{t} \mid t=-\tau, \ldots,-1,0,1, \ldots, \tau\right\}$ be a LTS, and an IVPLTS on $S$ can be defined as

$\widetilde{L}(p)=\left\{L^{k}\left[\gamma_{k}^{L}, \gamma_{k}^{U}\right] \mid L^{k} \in S, \quad 0 \leq \gamma_{k}^{L} \leq \gamma_{k}^{U} \leq 1, k=1,2, \ldots, \# \widetilde{L}(p)\right\}$,

where $L^{k}\left[\gamma_{k}^{L}, \gamma_{k}^{U}\right]$ is the linguistic term $L^{(k)}$ with respect to interval-valued probability $\left[\gamma_{k}^{L}, \gamma_{k}^{U}\right]$ with $\sum_{k=1}^{\# L(p)} \gamma_{k}^{U} \leq 1$.

The basic operational rules for IVPLTS can be seen as follows.

Definition 5 (see [7]). Let $S=\left\{s_{\alpha} \mid \alpha=-\tau, \ldots,-1,0,1, \ldots, \tau\right\}$ be a LTS, $\widetilde{L}_{1}(p)=\left\{L_{1}^{k}\left[\gamma_{1 k}^{L}, \gamma_{1 k}^{U}\right]\right\}$ and $\widetilde{L}_{2}(p)=\left\{L_{2}^{k}\left[\gamma_{2 k}^{L}, \gamma_{2 k}^{U}\right]\right\}$ be two IVPLTSs on $S, \xi$ be a positive number, $\eta_{1}^{(i)} \in g\left(\widetilde{L}_{1}(p)\right), \eta_{2}^{(j)} \in g\left(\widetilde{L}_{2}(p)\right), \quad i=1,2, \ldots, \# \widetilde{L}_{1}(p)$, $j=1,2, \ldots, \# \widetilde{L}_{2}(p)$, and $g:[-\tau, \tau] \longrightarrow[0,1]$ be an equivalent transformation function; then, the following rules hold:
(1) $\widetilde{L}_{1}(p) \oplus \widetilde{L}_{2}(p)=g^{-1}\left(\left\{\left(\eta_{1}^{(i)}+\eta_{2}^{(j)}-\eta_{1}^{(i)} \eta_{2}^{(j)}\right) \quad\left[\gamma_{1 i}^{L}\right.\right.\right.$. $\left.\left.\left.\gamma_{2 j}^{L}, \gamma_{1 i}^{U} \cdot \gamma_{2 j}^{U}\right]\right\}\right)$
(2) $\xi \widetilde{L}_{1}(p)=g^{-1}\left(\left\{\left(1-\left(1-\eta^{(i)}\right)^{\xi}\right)\left[\gamma_{1 i}^{L}, \gamma_{1 i}^{U}\right]\right\}\right)$

Bai et al. [7] proposed a normalization method to guarantee the ranges of probabilities in a standard interval $[0,1]$.

Let $\widetilde{L}(p)=\left\{L^{k}\left[\gamma_{k}^{L}, \gamma_{k}^{U}\right]\right\}$ be an IVPLTS; then, $\widetilde{L}(p)$ can be transformed to a standard IVPLTS $\bar{L}(p)=\left\{L^{k}\left[\gamma_{k}^{\bar{L}}, t \gamma_{k}^{\bar{U}}\right]\right\}$ as follows: 


$$
\begin{aligned}
\overline{\gamma_{k}^{L}} & =\frac{\gamma_{k}^{L}}{\sqrt{\sum_{k=1}^{\# \widetilde{L}(p)}\left(\gamma_{k}^{L}\right)^{2}+\left(\gamma_{k}^{U}\right)^{2}}}, \\
\gamma_{k}^{U} & =\frac{\gamma_{k}^{U}}{\sqrt{\sum_{k=1}^{\# \widetilde{L}(p)}\left(\gamma_{k}^{L}\right)^{2}+\left(\gamma_{k}^{U}\right)^{2}}} .
\end{aligned}
$$

For convenience narration, we still use $\widetilde{L}(p)=$ $\left\{L^{k}\left[\gamma_{k}^{L}, \gamma_{k}^{U}\right]\right\}$ as standard IVPLTS.

Jin et al. [10] proposed a distance measure as follows.

Definition $6 \quad$ (see [10]). Let $\widetilde{L}_{1}(p)=\left\{L_{1}^{k}\left[\begin{array}{ll}\gamma_{1 k}^{L}, & \gamma_{1 k}^{U}\end{array}\right]\right.$, $\left.k=1,2, \ldots, \# \widetilde{L}_{1}(p)\right\} \quad$ and $\quad \tilde{L}_{2}(p)=\left\{L_{2}^{k}\left[\gamma_{2 k}^{L}, \quad \gamma_{2 k}^{U}\right]\right.$, $\left.k=1,2, \ldots, \# \widetilde{L}_{2}(p)\right\}$ be two IVPLTSs, and then the distance between $\widetilde{L}_{1}(p)$ and $\widetilde{L}_{2}(p)$ can be defined as

$$
d\left(\widetilde{L}_{1}(p), \widetilde{L}_{2}(p)\right)=\frac{\sum_{k=1}^{\# \widetilde{L}_{1}(p)}(1 / 2)\left(\left|\gamma_{1 k}^{L} \times r_{1}^{k}-\gamma_{2 k}^{L} \times r_{2}^{k}\right|+\left|\gamma_{1 k}^{U} \times r_{1}^{k}-\gamma_{2 k}^{U} \times r_{2}^{k}\right|\right)}{\# \widetilde{L}_{1}(p)}
$$

where $r_{1}^{k}$ and $r_{2}^{k}$ are the subscripts of $L_{1}^{k}$ and $L_{2}^{k}$, respectively.

2.2. Regret Theory. Owing to the uncertain information, time pressure, and analysis capacity of DMs, in some cases, DMs usually have the feature of bounded rationality. Regret theory $[17,19,20]$ is a powerful tool to deal with this situation.

Given two alternatives $x_{1}$ and $x_{2}$, the perceived utility of DM for choosing $x_{1}$ can be computed as follows.

$$
U\left(x_{1}\right)=v\left(x_{1}\right)+R\left(v\left(x_{1}\right)-v\left(x_{2}\right)\right),
$$

where $v(\cdot)$ is a utility function satisfying $v^{\prime}(\cdot)>0$ and $v^{\prime \prime}(\cdot)<0$ and $R(\cdot)$ is a regret/rejoice function satisfying $R^{\prime}(\cdot)>0, R^{\prime \prime}(\cdot)<0$, and $R(0)=0$.

Zhang et al. [17] extended the RT theory from two alternatives to multiple ones.

Let $x_{1}, x_{2}, \ldots, x_{m}$ be $m$ alternatives and $x^{*}=$ $\max \left\{x_{i} \mid i t=n 1,2 q, h \ldots, x m\right\}$, and then perceived utility for $x_{i}$ can be obtained as

$$
U_{i}=v\left(x_{i}\right)+R\left(v\left(x_{i}\right)-v(x *)\right) .
$$

\section{A New Score Function for IVPLTS}

To compare different IVPLTSs, score function is a very useful tool. Pang et al. [3] proposed a score function for PLTS. Li and Wei [4] put forward a new score function based on D-S evidence theory. However, the research on score function for IVPLTS is infrequent. Therefore, we will propose a score function for IVPLTS.

Definition 7. Let $S=\left\{S_{t} \mid t=-\tau, \ldots,-1,0,1, \ldots, \tau\right\}$ be a LTS and $\widetilde{L}(p)=\left\{L^{k}\left[\gamma_{k}^{L}, \gamma_{k}^{U}\right]\right\}$ be an IVPLTS on $S$; then, the score function for $\widetilde{L}(p)$ can be defined as

$$
Q(\widetilde{L}(p))=\sum_{k=1}^{\# \widetilde{L}(p)}\left(\frac{\gamma_{k}^{L} \beta+\gamma_{k}^{U}(1-\beta)}{2}\right)\left(\frac{\tau+r^{k}}{2 \tau}\right)^{\theta}
$$

where $r^{k}$ is the subscript of $L^{k}, \beta \in[0,1]$ is a risk parameter, and $\theta>0$ is a preference parameter.

Note: the two parameters $\beta$ and $\theta$ can be obtained by decision makers according to their risk preferences. If the decision maker is risk-seeking, the two parameters can be set relatively large values. Conversely, if the decision maker is risk evading, they can be set relative small values.

Theorem 1. Let $\widetilde{L}(p)$ be a standard IVPLTS; then, score function $Q(\widetilde{L}(p))$ satisfies $0 \leq Q(\widetilde{L}(p)) \leq \#(\widetilde{L}(p))$.

Proof. Because $\widetilde{L}(p)$ is a standard IVPLTS, we can easily obtain that $\left[\gamma_{k}^{L}, \gamma_{k}^{U}\right] \subset[0,1]$ and $\sum_{k=1}^{\# \widetilde{L}(p)} \gamma_{k}^{L} \leq \sum_{k=1}^{\# \widetilde{L}(p)} \gamma_{k}^{U} \leq 1$. Because $\beta \in[0,1]$, we have $\gamma_{k}^{L} \leq\left(\gamma_{k}^{L} \beta+\gamma_{k}^{U}(1-\beta) / 2\right) \leq \gamma_{k}^{U}$. Then, we can draw a conclusion that $0 \leq \sum_{k=1}^{\# \widetilde{L}(p)}\left(\left(\gamma_{k}^{L} \beta+\gamma_{k}^{U}(1-\beta) / 2\right)\right) \leq \# \widetilde{L}(p) . \quad$ Because $-\tau \leq r^{k} \leq \tau$, we can obtain $0 \leq\left(\tau+r^{k} / 2 \tau\right) \leq 1$. Owing to $\theta>0$, we have $0 \leq\left(\tau+r^{k} / 2 \tau\right)^{\theta} \leq 1$. Therefore, we can obtain $0 \leq \sum_{k=1}^{\# \widetilde{L}(p)}\left(\gamma_{k}^{L} \beta+\gamma_{k}^{U}(1-\beta) / 2\right)\left(\left(\tau+r^{k}\right) / 2 \tau\right)^{\theta} \leq \# \widetilde{L}(p)$.

Theorem 2. For a fixed $\theta(\theta>0)$, the score function $Q(\widetilde{L}(p))$ for $\widetilde{L}(p)$ is a decreasing function of parameter $\beta(\beta>0)$.

Proof. Let $0<\beta_{1} \leq \beta_{2}$, and we can obtain $\gamma_{k}^{L} \beta_{1}+\gamma_{k}^{U}\left(1-\beta_{1}\right)=$ $\gamma_{k}^{U}+\left(\gamma_{k}^{L}-\gamma_{k}^{U}\right) \beta_{1}$ and $\gamma_{k}^{L} \beta_{2}+\gamma_{k}^{U}\left(1-\beta_{2}\right)=\gamma_{k}^{U}+\left(\gamma_{k}^{L}-\gamma_{k}^{U}\right) \beta_{2}$. Because $\gamma_{k}^{L}-\gamma_{k}^{U} \leq 0$, then we have $\left(\gamma_{k}^{L} \beta_{1}+\gamma_{k}^{U}(1-\right.$ $\left.\left.\beta_{1}\right) / 2\right) \geq\left(\gamma_{k}^{L} \beta_{2}+\gamma_{k}^{U}\left(1-\beta_{2}\right) / 2\right)$. Because $0 \leq\left(\tau+r^{k} / 2 \tau\right)^{\theta} \leq 1$ and $\theta>0$, we can obtain

$$
\sum_{k=1}^{\# \widetilde{L}(p)}\left(\frac{\gamma_{k}^{L} \beta_{1}+\gamma_{k}^{U}\left(1-\beta_{1}\right)}{2}\right)\left(\frac{\tau+r^{k}}{2 \tau}\right)^{\theta} \geq \sum_{k=1}^{\# \tilde{L}(p)}\left(\frac{\gamma_{k}^{L} \beta_{2}+\gamma_{k}^{U}\left(1-\beta_{2}\right)}{2}\right)\left(\frac{\tau+r^{k}}{2 \tau}\right)^{\theta} .
$$


Theorem 3. For a fixed $\beta(0 \leq \beta \leq 1)$, the score function $Q(\widetilde{L}(p))$ for $\widetilde{L}(p)$ is a decreasing function of parameter $\theta(0 \leq \theta \leq 1)$.

Proof. Assume that $0 \leq \theta_{1} \leq \theta_{2} \leq 1$. Because $0 \leq\left(\tau+r^{k} / 2 \tau\right) \leq$ 1 , we can easily obtain $0 \leq\left(\tau+r^{k} / 2 \tau\right)^{\theta_{2}} \leq\left(\tau+r^{k} / 2 \tau\right)^{\theta_{1}} \leq 1$. Because $0 \leq \beta \leq 1$, we can obtain $0 \leq\left(\gamma_{k}^{L} \beta+\gamma_{k}^{U}(1-\beta) / 2\right) \leq 1$ and

$$
\begin{gathered}
\sum_{k=1}^{\# \widetilde{L}(p)}\left(\frac{\gamma_{k}^{L} \beta+\gamma_{k}^{U}(1-\beta)}{2}\right)\left(\frac{\tau+r^{k}}{2 \tau}\right)^{\theta_{1}} \\
\geq \sum_{k=1}^{\# \widetilde{L}(p)}\left(\frac{\gamma_{k}^{L} \beta+\gamma_{k}^{U}(1-\beta)}{2}\right)\left(\frac{\tau+r^{k}}{2 \tau}\right)^{\theta_{2}} .
\end{gathered}
$$

Example 1. Let $S=\left\{s_{-3}, s_{-2}, s_{-1}, s_{0}, s_{1}, s_{2}, s_{3}\right\}$ be a LTS and $\widetilde{L}(p)=\left\{s_{0}[0.3,0.5], s_{1}[0.2,0.3], s_{2}[0.35,0.6]\right\}$ be an IVPLTS on $S$. Then, we can normalize $\widetilde{L}(p)$ as $\bar{L}(p)=\left\{s_{0}[0.31,0.51], s_{1}[0.20,0.31], s_{2}[0.36,0.61]\right\} \quad$ and obtain the score function as $Q(\bar{L}(P))=0.28$.

\section{A Novel IVPLTS Decision Method Based on Regret Theory and Cobweb Area Model}

4.1. Description of Decision-Making Problem. Given a decision-making problem, let $A=\left\{A_{1}, A_{2}, \ldots, A_{m}\right\}$ be an alternative set, $C=\left\{C_{1}, C_{2}, \ldots, C_{n}\right\}$ be a criterion set, and $W=\left(w_{1}, w_{2}, \ldots, w_{n}\right)$ be the criteria weights for criteria $C_{1}, C_{2}, \ldots, C_{n} \quad$ satisfying $\quad 0 \leq w_{j} \leq 1,(j=1,2, \ldots$, $n), \sum_{j=1}^{n} w_{j}=1$. DMs propose a decision matrix $X=\left(x_{i j}\right)_{m \times n}$, where $x_{i j}$ is a IVPLTS and indicates the value of alternative $A_{i}(i=1,2, \ldots, m)$ in terms of $C_{j}(j=$ $1,2, \ldots, n)$.

4.2. Obtaining the Criteria Weights Based on Maximizing Deviation Method. According to decision matrix $X=\left(x_{i j}\right)_{m \times n}$, we normalize $X$ to $Y=\left(y_{i j}\right)_{m \times n}$ based on equations (2) and (3). Based on equation (4), the deviation between alternative $A_{i}$ and other alternatives with respect to criterion $C_{j}$ can be computed as

$$
d_{i j}(w)=\sum_{l=1}^{m} d\left(y_{i j}, y_{l j}\right)
$$

Then, the deviation between all the alternatives and other ones with respect to criterion $C_{j}$ can be computed as

$$
d_{j}(w)=\sum_{i=1}^{m} \sum_{l=1}^{m} d\left(y_{i j}, y_{l j}\right)
$$

We establish a mathematical programming to obtain the criteria weights:

$$
\left\{\max d(w)=\sum_{j=1}^{n} \sum_{i=1}^{m} \sum_{l \neq i} w_{j} d\left(y_{i j}, y_{l j}\right), w_{j} \geq 0, j=1,2, \ldots, n, \quad \sum_{j=1}^{n} w_{j}^{2}=1 .\right.
$$

By solving (12), we can use the Lagrange function:

$$
L(w, \lambda)=\sum_{j=1}^{n} \sum_{i=1}^{m} \sum_{l \neq i} w_{j} d\left(y_{i j}, y_{l j}\right)+\frac{\lambda}{2}\left(\sum_{j=1}^{n} w_{j}^{2}-1\right) \text {, }
$$

where $\lambda$ is the Lagrange parameter.

Then, we use the following equations to obtain the criteria weights $w=\left(w_{1}, w_{2}, \ldots, w_{n}\right)$ :

$$
\left\{\frac{\partial L(w, \lambda)}{\partial w_{j}}=\sum_{i=1}^{m} \sum_{l \neq i} d\left(y_{i j}, y_{l j}\right)+\lambda w_{j}=0, \quad j=1,2, \ldots, n, \frac{\partial L(w, \lambda)}{\partial \lambda}=\sum_{j=1}^{n} w_{j}^{2}-1=0 .\right.
$$

Then, we can obtain the criteria weights $w=$ $\left(w_{1}, w_{2}, \ldots, w_{n}\right)$ as follows:

$$
w_{j}=\frac{\sum_{i=1}^{m} \sum_{l=1}^{m} d\left(y_{i j}, y_{l j}\right)}{\sum_{j=1}^{n} \sum_{i=1}^{m} \sum_{l=1}^{m} d\left(y_{i j}, y_{l j}\right)} .
$$

4.3. Computing Perceived Utility Based on Regret Theory. Based on equation (7), we can transform the standard IVPLTS decision matrix $Y=\left(y_{i j}\right)_{m \times n}$ into a score function matrix $Z=\left(z_{i j}\right)_{m \times n}$.
Let $z^{*}=\left(z_{1}^{*}, z_{2}^{*}, \ldots, z_{n}^{*}\right)$ be the reference points under the criteria $C_{1}, C_{2}, \ldots, C_{n}$, where $z_{j}^{*}=\max _{i}\left\{z_{i j}\right\}$ $(j=1,2, \ldots, n)$. In this paper, based on references $[19,21,22]$, we choose utility function $v(x)=x^{\alpha}$, where $\alpha$ is the risk aversion coefficient of the DMs satisfying $0<\alpha<1$. The smaller $\alpha$, the greater risk aversion of DMs. And regret/ rejoice function $R(\Delta v)=1-\exp (-\delta \Delta v)$, where $\delta$ is the regret aversion coefficient of the DMs satisfying $\delta>0$. The larger $\delta$, the larger regret aversion.

The graph for utility function $v(x)=x^{\alpha}$ with different $\alpha$ can be seen in Figure 1. 


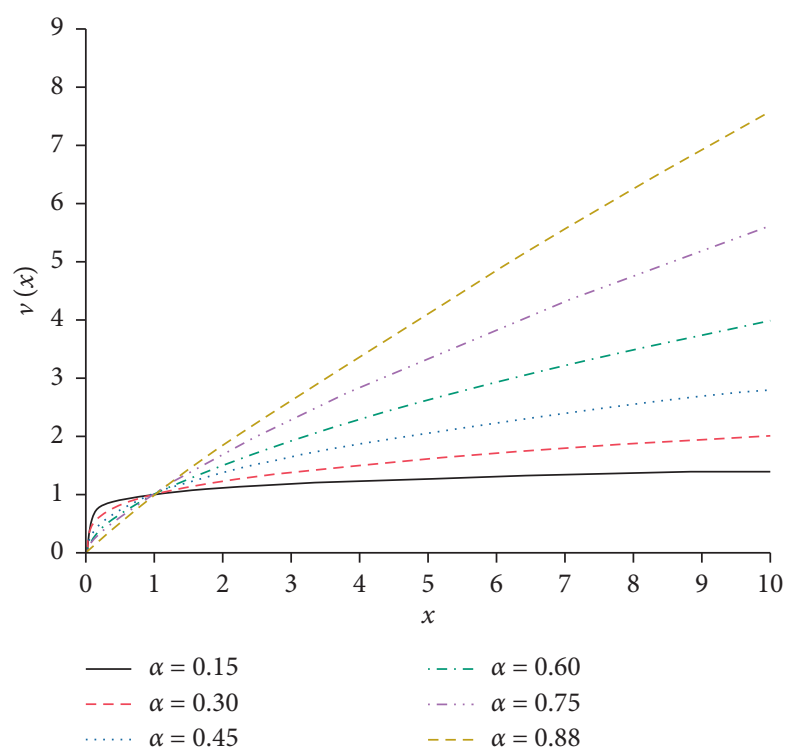

FIgURE 1: Graph for utility function with different $\alpha$.

The graph for regret/rejoice function $R(\Delta v)=$ $1-\exp (-\delta \Delta v)$ with different $\delta$ can be seen in Figure 2 .

Then, we can compute the perceived utility for $z_{i j}$ as

$$
U\left(z_{i j}\right)=v\left(z_{i j}\right)+R\left(v\left(z_{i j}\right)-v\left(z_{j}^{*}\right)\right) .
$$

In this paper, equation (16) can be transform to

$$
U\left(z_{i j}\right)=\left(z_{i j}\right)^{\alpha}+1-\exp \left(-\delta \cdot\left|\left(z_{i j}\right)^{\alpha}-\left(z_{j}^{*}\right)^{\alpha}\right|\right) \text {. }
$$

4.4. Aggregating Information Using Cobweb Area Model. Traditional method on aggregating information mainly focuses on the linear weighting model. In fact, in some cases, some extremely large or small values may make a very big impact on the final decision results [23]. To cope with this issue, we use the cobweb area model to aggregate information. The advantage of the cobweb area model lies in the fact that it uses the area of the values in different criteria and can reduce the influence of some extremely large or small values, which can partly solve the problem of malicious manipulation from some decision makers. The limitation of the cobweb area model is relatively massive calculation in the decision process.

The main idea of cobweb area model can be seen in Figure 3.

The main process of the cobweb area model is shown as follows:

(1) Determine the angles between the criteria $C_{1}, C_{2}, \ldots, C_{n}$ as $\xi_{1}=\xi_{2}=\cdots=\xi_{n}=360^{\circ} / n$.

(2) Compute the endpoint $s_{i j}=w_{j} U\left(z_{i j}\right)$ for alternative $A_{i}$ under the criterion $C_{j}(j=1,2, \ldots, n)$.

(3) Compute the cobweb area for alternative $A_{i}$ as

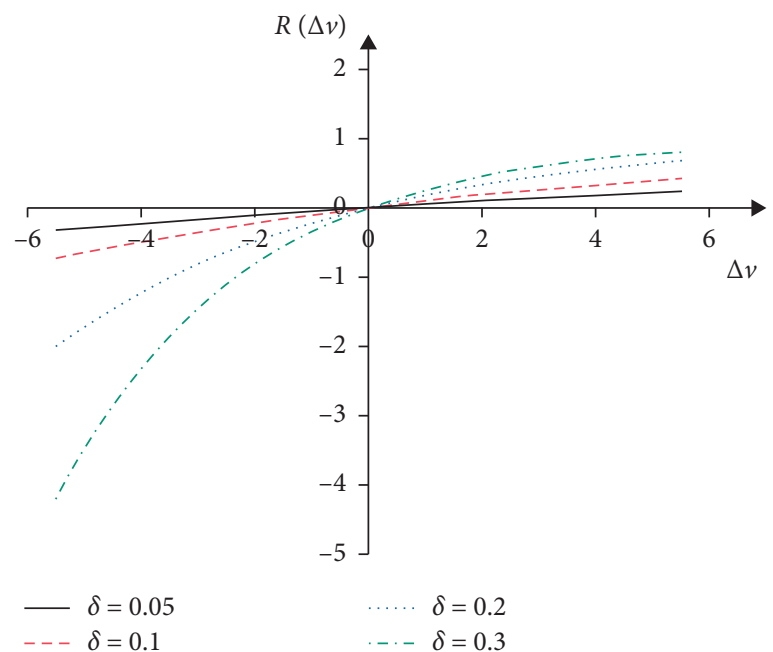

Figure 2: Graph for regret/rejoice function with different $\delta$.

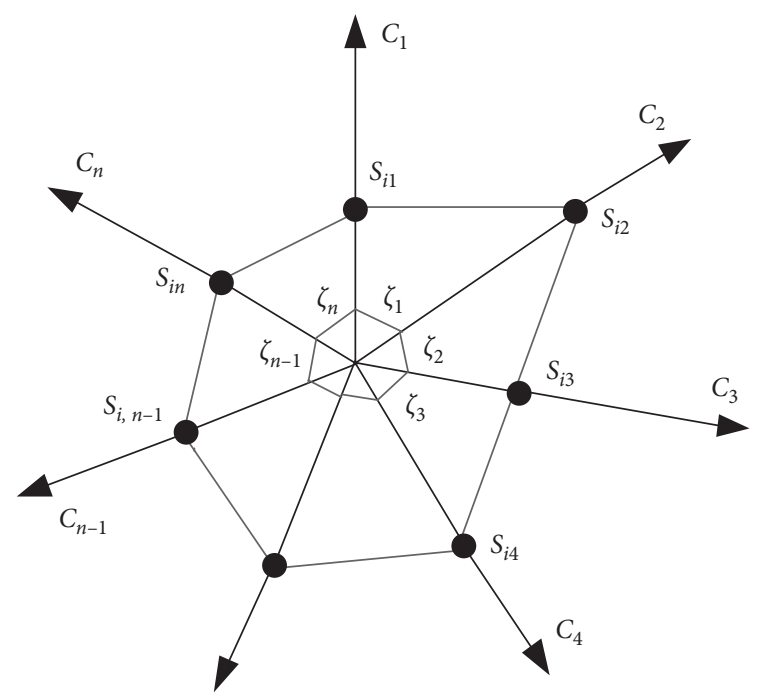

FIgURE 3: Main idea of the cobweb area model.

$$
\begin{aligned}
E_{i}= & \frac{1}{2} \times s_{i 1} \times s_{i 2} \times \sin \frac{360^{\circ}}{n}+\frac{1}{2} \times s_{i 2} \times s_{i 3} \times \sin \frac{360^{\circ}}{n} \\
& +\cdots+\frac{1}{2} \times s_{i n} \times s_{i 1} \times \sin \frac{360^{\circ}}{n} .
\end{aligned}
$$

(4) Rank the alternatives based on cobweb area. If $E_{i}>E_{k}$, then $A_{i}>A_{k}$.

Based on the above analysis, we can conclude the main decision steps as follows:

Step 1: based on decision matrix $X=\left(x_{i j}\right)_{m \times n}$, normalize $X$ to $Y=\left(y_{i j}\right)_{m \times n}$ based on equations (2) and (3) Step 2: according to equation (15), compute the criteria weights $W=\left(w_{1}, w_{2}, \ldots, w_{n}\right)$ 
Step 3: based on equation (7), we can transform the standard IVPLTS decision matrix $Y=\left(y_{i j}\right)_{m \times n}$ into a score function matrix $Z=\left(z_{i j}\right)_{m \times n}$

Step 4: compute the perceived utility $U\left(z_{i j}\right)$ based on equation (17)

Step 5: compute the cobweb area $E_{i}$ for alternative $A_{i}(i=1,2, \ldots, m)$ based on equation (18)

Step 6: rank the alternatives based on the value of cobweb area $E_{i}(i=1,2, \ldots, m)$

\section{Case Study}

With the development of medical level in China, the life expectancy of people has increased, and China has entered the aging society. By the end of 2017, there were more than $240,000,000$ elders (60 years old or over) in China. Longterm care for elders became a serious social problem. An effective way to deal with this problem is the combination of medical treatment and endowment. A comprehensive Grade $3 \mathrm{~A}$ hospital wants to cooperate with a nursing home based on the government policy. After preliminary screening, there are four nursing homes (alternatives) $A_{1}, A_{2}, A_{3}, A_{4}$ shortlisted. Some experts from hospitals, government, and nursing homes evaluate the four alternatives according to four factors (criteria) $C_{1}$ (service level), $C_{2}$ (business performance), $C_{3}$ (hardware facilities), and $C_{4}$ (management level). The experts use the LTS:

$$
S=\left\{s_{-3}: \text { extremely poor, } s_{-2}: \text { very poor, } s_{-1}: \text { poor, } s_{0}: \text { medium, } s_{1}: \text { good, } s_{2}: \text { very good, } s_{3}: \text { extremely good }\right\}
$$

to express their opinions.

The experts give their opinions and propose a decision matrix $X$ as Table 1 .

For example, the linguistic assessment of $A_{1}$ is with respect to $C_{1}$ and the experts evaluate the probability of $s_{-1}$ as $0.5-0.7$ and the probability of $s_{0}$ as $0.2-0.4$.

\subsection{Decision Process}

Step 1: based on equations (2) and (3), we can transform the decision matrix $X$ to normalized matrix $Y$, as shown in Table 2.

Step 2: according to equation (15), we can obtain the criteria weights:

$$
\begin{aligned}
& w_{1}=0.289, \\
& w_{2}=0.254, \\
& w_{3}=0.182, \\
& w_{4}=0.275 .
\end{aligned}
$$

Step 3: based on equation (7) and matrix $Y$, we can obtain the score function matrix $Z$, as shown in Table 3 $(\beta=0.5, \theta=1)$.

Step 4: based on equation (17), we can obtain the values of perceived utility $U\left(z_{i j}\right)$, as shown in Table 4 $(\alpha=0.88$ and $\delta=0.3$, see [21]).

Step 5: based on equation (18), we can compute the cobweb area $E_{i}$ for alternative $A_{i}(i=1,2, \ldots, 4)$ as follows:

$$
\begin{aligned}
E_{1}= & \frac{1}{2} \times 0.289 \times 0.34 \times 0.99 \times 1+\frac{1}{2} \times 0.254 \times 0.99 \times 1.21 \\
& \times 1+\frac{1}{2} \times 0.182 \times 0.1 .21 \times 0.93 \times 1+\frac{1}{2} \times 0.275 \times 0.93 \\
& \times 0.34 \times 1=0.356 .
\end{aligned}
$$

Similarly, we can obtain $E_{2}=1.044, E_{3}=$ 0.882 , and $E_{4}=1.593$.

Step 6: the ranking result is $A_{4}>A_{2}>A_{3}>A_{1}$.

Based on the selection of the above shortlisted nursing homes constructed in this paper, the ranking result is $A_{4}>A_{2}>A_{3}>A_{1}$. In this case, the parameters can be selected by the decision maker according to their preferences. When the decision maker is optimistic about the decision problem, we can select $0.5<\beta \leq 1$. When the decision maker is eclectic, we can select $\beta=0.5$. When the decision maker is pessimistic, we can select $0 \leq \beta<0.5$. The parameter $\theta$ reflects the preference of value judgment. If the decision maker is radical, we can select $\theta>1$. Similarly, we can select $0<\theta \leq 1$ if the decision maker is conservative. According to the values of the criteria weight, it can be seen that $C_{1}$ (service level) is the most important criterion. So, managers should focus on improving service levels.

5.2. Comparative Analysis. In this section, we will compare our method with the TOPSIS method for IVPLTS proposed by [10] and the VIKOR method proposed by [11].

5.2.1. Comparison with the TOPSIS Method. The main decision process of the method proposed by [10] can be concluded as the following decision steps:

(1) Determine the positive ideal solution PIS $Y^{*}=\left(y_{1}^{*}, y_{2}^{*}, \ldots, y_{n}^{*}\right)$ and negative ideal solution NIS $Y^{-}=\left(y_{1}^{-}, y_{2}^{-}, \ldots, y_{n}^{-}\right)$

(2) Compute the deviation degree between every alternative $A_{i}$ and PIS $Y^{*}=\left(y_{1}^{*}, y_{2}^{*}, \ldots, y_{n}^{*}\right)$ as $d_{i}^{+}=d\left(A_{i}, Y^{*}\right)$

(3) Compute the deviation degree between every alternative $A_{i}$ and NIS $Y^{-}=\left(y_{1}^{-}, y_{2}^{-}, \ldots, y_{n}^{-}\right)$as $d_{i}^{-}=d\left(A_{i}, Y^{-}\right)$

(4) Compute the closeness coefficient $\mathrm{CI}\left(A_{i}\right)=$ $\left(d_{i}^{-} / \max _{i}\left\{d_{i}^{-}\right\}\right)-\left(d_{i}^{+} / \min _{i}\left\{d_{i}^{+}\right\}\right)$ 
Table 1: Decision matrix $X$ proposed by experts.

\begin{tabular}{|c|c|c|c|c|}
\hline & $C_{1}$ & $C_{2}$ & $C_{3}$ & $C_{4}$ \\
\hline$A_{1}$ & $\left\{\begin{array}{c}s_{-1}[0.5,0.7], \\
s_{0}[0.2,0.4],\end{array}\right\}$ & $\left\{\begin{array}{l}s_{1}[0.3,0.6], \\
s_{2}[0.2,0.3],\end{array}\right\}$ & $\left.\begin{array}{l}s_{1}[0.5,0.6] \\
s_{2}[0.2,0.5]\end{array}\right\}$ & $\left\{\begin{array}{c}s_{-1}[0.4,0.6] \\
s_{0}[0.4,0.7]\end{array}\right.$ \\
\hline$A_{2}$ & $\left\{\begin{array}{l}s_{0}[0.48,0.7], \\
s_{1}[0.55,0.8],\end{array}\right\}$ & $\left\{\begin{array}{l}s_{1}[0.5,0.8], \\
s_{2}[0.3,0.5],\end{array}\right\}$ & $\left.\begin{array}{l}s_{2}[0.5,0.7] \\
s_{3}[0.3,0.4]\end{array}\right\}$ & $\left\{\begin{array}{l}s_{1}[0.35,0.5] \\
s_{2}[0.4,0.55]\end{array}\right.$ \\
\hline$A_{3}$ & $\begin{array}{c}s_{-1}[0.45,0.6] \\
s_{0}[0.55,0.7]\end{array}$ & $\left\{\begin{array}{l}s_{2}[0.5,0.6], \\
s_{3}[0.4,0.6],\end{array}\right\}$ & $\left.\begin{array}{l}s_{2}[0.6,0.9], \\
s_{3}[0.2,0.4],\end{array}\right\}$ & $\left\{\begin{array}{l}s_{0}[0.3,0.6], \\
s_{1}[0.6,0.7],\end{array}\right\}$ \\
\hline$A_{4}$ & $\left\{\begin{array}{l}s_{1}[0.3,0.6], \\
s_{2}[0.5,0.8],\end{array}\right\}$ & $\left.\begin{array}{l}s_{0}[0.4,0.55] \\
s_{1}[0.55,0.7]\end{array}\right\}$ & $\left.\begin{array}{l}s_{2}[0.3,0.6], \\
s_{3}[0.2,0.5],\end{array}\right\}$ & $\left\{\begin{array}{l}s_{1}[0.4,0.5], \\
s_{2}[0.5,0.6] .\end{array}\right\}$ \\
\hline
\end{tabular}

TABle 2: Normalized decision matrix $Y$.

\begin{tabular}{|c|c|c|c|c|}
\hline & $C_{1}$ & $C_{2}$ & $C_{3}$ & $C_{4}$ \\
\hline$A_{1}$ & $\left.\begin{array}{c}s_{-1}[0.52,0.72] \\
s_{0}[0.21,0.41]\end{array}\right\}$ & $\begin{array}{l}s_{1}[0.39,0.79] \\
s_{2}[0.26,0.39]\end{array}$ & $\left.\begin{array}{l}s_{1}[0.53,0.63] \\
s_{2}[0.21,0.53]\end{array}\right\}$ & $\begin{array}{c}s_{-1}[0.37,0.55] \\
s_{0}[0.37,0.65]\end{array}$ \\
\hline$A_{2}$ & $\left\{\begin{array}{l}s_{0}[0.37,0.54], \\
s_{1}[0.43,0.62],\end{array}\right\}$ & $\left.\begin{array}{l}s_{1}[0.45,0.72] \\
s_{2}[0.27,0.45]\end{array}\right\}$ & $\left.\begin{array}{l}s_{2}[0.50,0.70] \\
s_{3}[0.30,0.40]\end{array}\right\}$ & $\left\{\begin{array}{l}s_{1}[0.38,0.55] \\
s_{2}[0.44,0.60]\end{array}\right\}$ \\
\hline$A_{3}$ & $\begin{array}{c}s_{-1}[0.39,0.52] \\
s_{0}[0.47,0.60]\end{array}$ & $\left.\begin{array}{l}s_{2}[0.47,0.56], \\
s_{3}[0.38,0.56],\end{array}\right\}$ & $\left.\begin{array}{l}s_{2}[0.51,0.77] \\
s_{3}[0.17,0.34]\end{array}\right\}$ & $\left\{\begin{array}{l}s_{0}[0.26,0.53] \\
s_{1}[0.53,0.61]\end{array}\right\}$ \\
\hline$A_{4}$ & $\left\{\begin{array}{l}s_{1}[0.26,0.52] \\
s_{2}[0.43,0.69]\end{array}\right.$ & $\begin{array}{l}s_{0}[0.36,0.49] \\
s_{1}[0.49,0.63]\end{array}$ & $\begin{array}{l}s_{2}[0.35,0.70], \\
s_{3}[0.23,0.58],\end{array}$ & $\left\{\begin{array}{l}s_{1}[0.40,0.50] \\
s_{2}[0.50,0.59]\end{array}\right\}$ \\
\hline
\end{tabular}

TABLE 3: Score function matrix $Z$.

\begin{tabular}{ccccc}
\hline & $C_{1}$ & $C_{2}$ & $C_{3}$ & $C_{4}$ \\
\hline$A_{1}$ & 0.74 & 1.28 & 1.44 & 1.20 \\
$A_{2}$ & 1.63 & 1.41 & 1.64 & 2.02 \\
$A_{3}$ & 1.27 & 2.18 & 1.19 & 1.77 \\
$A_{4}$ & 2.16 & 1.74 & 1.88 & 2.10 \\
\hline
\end{tabular}

(5) Rank the alternatives according to CI $\left(A_{i}\right)$

We use the TOPSIS method proposed by [10] to solve our decision problem.

(1) We can obtain the PIS and NIS, as shown in Table 5.

(2) We can obtain the deviation degrees between every alternative $A_{i} \quad(i=1,2,3,4)$ and PIS $Y^{*}=$ $\left(y_{1}^{*}, y_{2}^{*}, \ldots, y_{4}^{*}\right)$ as

$$
\begin{aligned}
d_{1}^{+} & =0.821, \\
d_{2}^{+} & =0.349, \\
d_{3}^{+} & =0.479, \\
d_{4}^{+} & =0.240, \\
\min _{1 \leq i \leq m} d_{i}^{+} & =0.240 .
\end{aligned}
$$

TABLE 4: Values of perceived utility.

\begin{tabular}{ccccc}
\hline & $C_{1}$ & $C_{2}$ & $C_{3}$ & $C_{4}$ \\
\hline$A_{1}$ & 0.34 & 0.99 & 1.26 & 0.93 \\
$A_{2}$ & 1.40 & 1.14 & 1.48 & 1.83 \\
$A_{3}$ & 0.99 & 1.98 & 0.98 & 1.57 \\
$A_{4}$ & 1.97 & 1.51 & 1.75 & 1.92 \\
\hline
\end{tabular}

(3) The deviation degree between every alternative $A_{i}$ and NIS $Y^{-}=\left(y_{1}^{-}, y_{2}^{-}, \ldots, y_{4}^{-}\right)$can be obtained as

$$
\begin{aligned}
d_{1}^{-} & =0.069, \\
d_{2}^{-} & =0.485, \\
d_{3}^{-} & =0.318, \\
d_{4}^{-} & =0.732, \\
\max _{1 \leq i \leq m} d_{i}^{-} & =0.732 .
\end{aligned}
$$

(4) We can compute the closeness coefficient as

$$
\begin{aligned}
& \mathrm{CI}\left(x_{1}\right)=-2.421, \\
& \mathrm{CI}\left(x_{2}\right)=-0.455, \\
& \mathrm{CI}\left(x_{3}\right)=-0.992, \\
& \mathrm{CI}\left(x_{4}\right)=0 .
\end{aligned}
$$


TABle 5: PIS and NIS for decision matrix $Y$.

\begin{tabular}{|c|c|c|c|c|}
\hline & $C_{1}$ & $C_{2}$ & $C_{3}$ & $C_{4}$ \\
\hline PIS & $\left\{\begin{array}{l}s_{1}[0.26,0.52], \\
s_{2}[0.43,0.69],\end{array}\right\}$ & $\left\{\begin{array}{l}s_{2}[0.47,0.56], \\
s_{3}[0.38,0.56],\end{array}\right\}$ & $\left\{\begin{array}{l}s_{2}[0.35,0.70], \\
s_{3}[0.23,0.58],\end{array}\right\}$ & $\left\{\begin{array}{l}s_{1}[0.40,0.50], \\
s_{2}[0.50,0.59],\end{array}\right\}$ \\
\hline NIS & $\left\{\begin{array}{c}s_{-1}[0.52,0.72], \\
s_{0}[0.21,0.41],\end{array}\right\}$ & $\left\{\begin{array}{l}s_{1}[0.39,0.79], \\
s_{2}[0.26,0.39],\end{array}\right\}$ & $\left\{\begin{array}{l}s_{2}[0.51,0.77], \\
s_{3}[0.17,0.34],\end{array}\right\}$ & $\left\{\begin{array}{c}s_{-1}[0.37,0.55] \\
s_{0}[0.37,0.65]\end{array}\right.$ \\
\hline
\end{tabular}

(5) The ranking result is $A_{4}>A_{2}>A_{3}>A_{1}$.

5.2.2. Comparison with the VIKOR Method. To further verify our method, IVPLTS-based VIKOR proposed by [11] is taken for comparison with our method. The main decision process of the method can be concluded as the following decision steps:

(1) Determine the positive ideal solution (PIS) and negative ideal solution (NIS):

$$
\begin{array}{r}
\widetilde{L}^{*}(p)=\max _{j \in \text { benefit }}\left(\frac{\left(r_{i j}^{k} \gamma_{k}^{L}+r_{i j}^{k} \gamma_{k}^{U}\right)}{2}\right), \\
\quad \operatorname{ormin}_{j \in \operatorname{cost}}\left(\frac{\left(r_{i j}^{k} \gamma_{k}^{L}+r_{i j}^{k} \gamma_{k}^{U}\right)}{2}\right), \\
\widetilde{L}^{-}(p)=\min _{j \in \text { benefit }}\left(\frac{\left(r_{i j}^{k} \gamma_{k}^{L}+r_{i j}^{k} \gamma_{k}^{U}\right)}{2}\right), \\
\operatorname{or~max~}_{j \in \operatorname{cost}}\left(\frac{\left(r_{i j}^{k} \gamma_{k}^{L}+r_{i j}^{k} \gamma_{k}^{U}\right)}{2}\right) .
\end{array}
$$

(2) Compute the group utility $(S)$ and individual regret $(R)$ of every alternative by using the following equations:

$$
\begin{aligned}
& S^{l}=\left(\sum_{j \in \text { benefit }} w_{j}\left(\frac{d\left(\widetilde{L}\left(\gamma_{k}^{U}\right), \widetilde{L}^{*}(p)\right)}{d\left(\widetilde{L}^{*}(p), \widetilde{L}^{-}(p)\right)}\right)+\sum_{j \in \text { cost }} w_{j}\left(\frac{d\left(\widetilde{L}\left(\gamma_{k}^{L}\right), \widetilde{L}^{*}(p)\right)}{d\left(\widetilde{L}^{*}(p), \widetilde{L}^{-}(p)\right)}\right)\right), \\
& S^{u}=\left(\sum_{j \in \text { benefit }} w_{j}\left(\frac{d\left(\widetilde{L}\left(\gamma_{k}^{L}\right), \widetilde{L}^{*}(p)\right)}{d\left(\widetilde{L}^{*}(p), \widetilde{L}^{-}(p)\right)}\right)+\sum_{j \in \operatorname{cost}} w_{j}\left(\frac{d\left(\widetilde{L}\left(\gamma_{k}^{U}\right), \widetilde{L}^{*}(p)\right)}{d\left(\widetilde{L}^{*}(p), \widetilde{L}^{-}(p)\right)}\right)\right), \\
& R^{l}=\max \left(w_{j}\left(\frac{d\left(\widetilde{L}\left(\gamma_{k}^{U}\right), \widetilde{L}^{*}(p)\right)}{d\left(\widetilde{L}^{*}(p), \widetilde{L}^{-}(p)\right)}\right), w_{j}\left(\frac{d\left(\widetilde{L}\left(\gamma_{k}^{L}\right), \widetilde{L}^{*}(p)\right)}{\left.d\left(\widetilde{L}^{*}(p), \widetilde{L}^{-}(p)\right)\right)}\right),\right. \\
& R^{u}=\max \left(w_{j}\left(\frac{d\left(\widetilde{L}\left(\gamma_{k}^{L}\right), \widetilde{L}^{*}(p)\right)}{d\left(\widetilde{L}^{*}(p), \widetilde{L}^{-}(p)\right)}\right), w_{j}\left(\frac{d\left(\widetilde{L}\left(\gamma_{k}^{U}\right), \widetilde{L}^{*}(p)\right)}{d\left(\widetilde{L}^{*}(p), \widetilde{L}^{-}(p)\right)}\right)\right) .
\end{aligned}
$$

(3) Compute the merit function $(Q)$ for alternatives, respectively, by using the following equations:

$$
\begin{aligned}
& Q^{l}=v\left(\frac{S^{l}-S^{*}}{S^{-}-S^{*}}\right)+(1-v)\left(\frac{R^{l}-R^{*}}{R^{-}-R^{*}}\right), \\
& Q^{u}=v\left(\frac{S^{u}-S^{*}}{S^{-}-S^{*}}\right)+(1-v)\left(\frac{R^{u}-R^{*}}{R^{-}-R^{*}}\right),
\end{aligned}
$$

where $\left[Q^{l}, Q^{u}\right]$ is the interval range of merit function, $v \in[0,1]$ represents the strategy of the decision makers and $v \in[0,1]$, $S^{*}=\min \left(S^{l}\right), R^{*}=\min \left(R^{l}\right), S^{-}=\max \left(S^{u}\right)$, and $R^{-}=\max$ $\left(R^{u}\right)$.

(4) Determine the final merit function $(Q)$ by calculating the mean of $Q^{l}$ and $Q^{u}$. If $Q_{i}>Q_{k}$, then $A_{i}<A_{k}$.
We use the VIKOR method proposed by [11] to solve our decision problem and draw the ranking results in Table 6.

It is obvious that the decision results between our method and the one proposed by [10] are coincident. And it is different between our method and the one proposed by [11] in most cases. When $v=0.9$ and $v=1$, the results of the two methods are the same. Compared with the methods proposed by $[10,11]$, our method has the following traits:

(1) We use the regret theory to solve the decision problem, which will reflect the DMs' bounded rationality and make the decision result reasonable.

(2) We utilize the cobweb area model to aggregate decision information rather than traditional linear weighting method, which will overcome the problem 
TABLE 6: Ranking results for the IVPLTS-VIKOR method.

\begin{tabular}{lccccc}
\hline & \multicolumn{5}{c}{ Merit function $(Q)$} \\
& $A_{1}$ & $A_{2}$ & $A_{3}$ & $A_{4}$ & Ranking order \\
\hline 0.1 & 0.9055 & 0.1779 & 0.7991 & 0.5858 & $A_{2}>A_{4}>A_{3}>A_{1}$ \\
0.2 & 0.8699 & 0.1668 & 0.7292 & 0.5235 & $A_{2}>A_{4}>A_{3}>A_{1}$ \\
0.3 & 0.8342 & 0.1557 & 0.6593 & 0.4612 & $A_{2}>A_{4}>A_{3}>A_{1}$ \\
0.4 & 0.7986 & 0.1447 & 0.5894 & 0.3990 & $A_{2}>A_{4}>A_{3}>A_{1}$ \\
0.5 & 0.7630 & 0.1336 & 0.5195 & 0.3367 & $A_{2}>A_{4}>A_{3}>A_{1}$ \\
0.6 & 0.7274 & 0.1226 & 0.4496 & 0.2744 & $A_{2}>A_{4}>A_{3}>A_{1}$ \\
0.7 & 0.6918 & 0.1115 & 0.3797 & 0.2122 & $A_{2}>A_{4}>A_{3}>A_{1}$ \\
0.8 & 0.6561 & 0.1005 & 0.3098 & 0.1499 & $A_{2}>A_{4}>A_{3}>A_{1}$ \\
0.9 & 0.6205 & 0.0894 & 0.2399 & 0.0876 & $A_{4}>A_{2}>A_{3}>A_{1}$ \\
1 & 0.5849 & 0.0784 & 0.1700 & 0.0254 & $A_{4}>A_{2}>A_{3}>A_{1}$ \\
\hline
\end{tabular}

that some extremely large or small values may intensely influence the final decision results.

\section{Conclusions}

In this paper, we put forward a new decision method for IVPLTS based on regret theory. First, we propose a new score function for IVPLTS to transform the IVPLTSs into crisp numbers. We verify three mathematical properties of the score function, and we can see that the score function has great flexibility by adjusting the two parameters. Then, we use the regret theory to obtain the DMs' perceived utilities. Furthermore, we propose a cobweb area model to aggregate decision information to alleviate the problem that some extremely large or small values may exert an influence in final decision result. Finally, we apply our method to a real case of evaluating nursing homes and compare our method with the traditional TOPSIS method and VIKOR method. The comparative analysis illustrates the effectiveness and traits of our method.

Future research mainly focuses on multiple stages decision-making method for IVPLTSs.

\section{Data Availability}

All the data used to support the study are available from the corresponding author upon request.

\section{Conflicts of Interest}

The authors have no conflicts of interest.

\section{Acknowledgments}

This research was funded by Humanities and Social Science Fund of Ministry of Education of China, Grant no. 19YJA630039.

\section{References}

[1] L. A. Zadeh, "The concept of a linguistic variable and its application to approximate reasoning-I," Information Sciences, vol. 8, no. 3, pp. 199-249, 1975.

[2] R. M. Rodriguez, L. Martinez, and F. Herrera, "Hesitant fuzzy linguistic term sets for decision making," IEEE Transactions on Fuzzy Systems, vol. 20, no. 1, pp. 109-119, 2012.
[3] Q. Pang, H. Wang, and Z. Xu, "Probabilistic linguistic term sets in multi-attribute group decision making," Information Sciences, vol. 369, pp. 128-143, 2016.

[4] P. Li and C. P. Wei, "An emergency decision-making method based on D-S evidence theory for probabilistic linguistic term sets," International Journal of Disaster Risk Reduction, vol. 37, 2019.

[5] X. Yu, H. Chen, and Z. Ji, "Combination of probabilistic linguistic term sets and promethee to evaluate meteorological disaster risk: case study of Southeastern China," Sustainability, vol. 11, no. 5, p. 1405, 2019.

[6] Y. Zhang, Z. Xu, H. Wang, and H. Liao, "Consistency-based risk assessment with probabilistic linguistic preference relation," Applied Soft Computing, vol. 49, pp. 817-833, 2016.

[7] C. Bai, R. Zhang, S. Shen, C. Huang, and X. Fan, "Intervalvalued probabilistic linguistic term sets in multi-criteria group decision making," International Journal of Intelligent Systems, vol. 33, no. 6, pp. 1301-1321, 2018.

[8] W. Yu, H. Zhang, and B. Li, "Comparison and operators based on uncertain probabilistic linguistic term set," Journal of Intelligent \& Fuzzy Systems, vol. 36, no. 6, pp. 6359-6379, 2019.

[9] S. Zhang, Z. Xu, and H. Wu, "Fusions and preference relations based on probabilistic interval-valued hesitant fuzzy information in group decision making," Soft Computing, vol. 23, no. 17 , pp. 8291-8306, 2019.

[10] C. Jin, H. Wang, and Z. Xu, "Uncertain probabilistic linguistic term sets in group decision making," International Journal of Fuzzy Systems, vol. 21, no. 4, pp. 1241-1258, 2019.

[11] R. Krishankumar, A. R. Mishra, K. S. Ravichandran et al., "A group decision framework for renewable energy source selection under interval-valued probabilistic linguistic term set," Energies, vol. 13, no. 4, 2020.

[12] P. Liu and Y. Li, "An extended MULTIMOORA method for probabilistic linguistic multi-criteria group decision-making based on prospect theory," Computers \& Industrial Engineering, vol. 136, pp. 528-545, 2019.

[13] J. Gu, Y. Zheng, X. L. Tian, and Z. S. Xu, "A decision-making framework based on prospect theory with probabilistic linguistic term sets," Journal of the Operational Research Society, vol. 4, pp. 1-10, 2020.

[14] L. Wang, Y. P. Hu, H. C. Liu, and H. Shi, "A linguistic risk prioritization approach for failure mode and effects analysis: a case study of medical product development," Quality and Reliability Engineering International, vol. 35, no. 6, pp. 1735-1752, 2019.

[15] J. Qin, "Regret-based capital asset pricing model," Journal of Banking \& Finance, vol. 114, 2020.

[16] C. Bai and J. Sarkis, "A supply chain transparency and sustainability technology appraisal model for blockchain technology," International Journal of Production Research, vol. 58, no. 7, pp. 2142-2162, 2020.

[17] S. Zhang, J. Zhu, X. Liu, and Y. Chen, "Regret theory-based group decision-making with multidimensional preference and incomplete weight information," Information Fusion, vol. 31, pp. 1-13, 2016.

[18] Z. Xu, "Deviation measures of linguistic preference relations in group decision making," Omega, vol. 33, no. 3, pp. 249-254, 2005.

[19] D. E. Bell, "Regret in decision making under uncertainty," Operations Research, vol. 30, no. 5, pp. 961-981, 1982.

[20] G. Loomes and R. Sugden, "Regret theory: an alternative theory of rational choice under uncertainty," The Economic Journal, vol. 92, no. 368, pp. 805-824, 1982. 
[21] A. Tversky and D. Kahneman, "Advances in prospect theory: cumulative representation of uncertainty," Journal of Risk and Uncertainty, vol. 5, no. 4, pp. 297-323, 1992.

[22] C. G. Chorus, "Regret theory-based route choices and traffic equilibria," Transportmetrica, vol. 8, no. 4, pp. 291-305, 2010.

[23] P. Li and C. P. Wei, "A novel grey target decision method based on a cobweb area model for standard interval grey numbers," Journal of Grey System, vol. 31, no. 3, pp. 29-44, 2019. 